\title{
Inhibitory Effect of Semen Litchi Drug Serum on the Proliferation of Human Hepatoma HepG2 Cells and Expression of VEGF and MMP-9
}

\author{
Lichun Zhao, Peiliang Yu, Tao Yang, Gailian Zhou and Nong Tang
}

\begin{abstract}
Objective: To investigate inhibitory effect of semen litchi drug serum on proliferation of human hepatoma HepG2 cells and its effect on the expression of vascular endothelial growth factor (VEGF) and matrix metalloproteinase-9 (MMP-9).

Study Design: An experimental study.

Place and Duration of Study: College of Pharmacy, Guangxi University of Chinese Medicine, China, from June 2017 to January 2018.

Methodology: Semen litchi drug serum with concentrations of $0 \mathrm{mg} / \mathrm{kg}$ (control group), $3 \mathrm{~g} / \mathrm{kg}$ (low dose group), $6 \mathrm{~g} / \mathrm{kg}$ (medium dose group) and $12 \mathrm{~g} / \mathrm{kg}$ (high dose group) was used to act on HepG2 cells at the logarithmic phase. Inhibitory effect of semen litchi drug serum on cell growth, expression of VEGF and MMP-9 mRNA and protein was detected.

Results: Inhibitory effect of semen litchi drug serum on the proliferation of HepG2 cells significantly increased with the increase of drug concentration, which was dose-time dependent. Expression levels of VEGF and MMP-9 mRNA in HepG2 cells after 48 hours of treatment by semen litchi low-dose group, medium-dose group, and high-dose group were lower than those in control group (all $p<0.001$ ). After acting on HepG2 cells for 48 hours, relative expressions of VEGF and MMP-9 protein in semen litchi low-dose group, medium-dose group, and high-dose group were lower than those in control group (all $p<0.001$ ).

Conclusion: Semen litchi drug serum can inhibit proliferation of hepatoma cells in vitro. The anti-hepatoma effect of semen litchi drug serum may be exerted through down-regulating the expression of VEGF and MMP-9 and inhibiting angiogenesis of hepatocellular carcinoma.
\end{abstract}

Key Words: Litchi, Serum pharmacology, Hepatocellular carcinoma, Cell proliferation, VEGF, MMP-9.

\section{INTRODUCTION}

Liver cancer is a common malignant tumor. Early surgical resection is the key to its treatment, but most patients are already at the advanced stage when diagnosed.1,2 The overall curative effect is poor.3,4 Therefore, finding new anti-hepatoma drugs has become a research focus in the treatment of liver cancer. Semen litchi is a dry mature seed of litchi, which belongs to Sapindaceae. It is sweet, slightly bitter, and has the effects of promoting the circulation of blood dissolving lumps, resisting coldness and relieving pain. Modern research showed that semen litchi had many pharmacological effects, such as blood sugar reduction, blood lipid regulation, liver protection and anti-oxidation. 5,6

The rapid growth of cancer tissue has to be accompanied by the growth of new blood vessels so as to ensure sufficient nutrient supply and maintain the exuberant

College of Pharmacy, Guangxi University of Chinese Medicine, Nanning, 530200, China

Correspondence: Dr. Nong Tang, College of Pharmacy, Guangxi University of Chinese Medicine, Nanning, 530200, China

E-mail:bfvbpm56@163.com

Received: July 04, 2018; Accepted: December 20, 2018 metabolism of liver cancer cells and their metastatic spread. Therefore, it is an important anticancer strategy to inhibit the formation of blood vessels in liver cancer and cut off the life-blood of liver cancer growth and metastasis. Experiments have shown that vascular endothelial growth factor (VEGF) is expressed strongly in hepatoma cells. ${ }^{7}$

VEGF is the most well-known cytokine that promotes vascular endothelial growth. The expression of matrix metalloproteinase-9 (MMP-9) in hepatocellular carcinoma is significantly higher than that in adjacent tissues, and the imbalance of MMP-9 expression is closely related to the invasion and metastasis of hepatocellular carcinoma. ${ }^{8}$ At present, there are few studies on the effect of semen litchi drug serum on the expression of VEGF and MMP-9 in human hepatoma HepG2 cells.

The objective of this study was to investigate inhibitory effect of semen litchi drug serum on proliferation of human hepatoma HepG2 cells and its effect on the expression of VEGF and MMP-9, and to analyse the possible mechanism of anti-hepatoma by semen litchi drug serum.

\section{METHODOLOGY}

This study was conducted at the College of Pharmacy, Guangxi University of Chinese Medicine, China, from 
June 2017 to January 2018. The research was conducted after approval from the Committee on Animal Research and Ethics. Eight healthy male New Zealand rabbits weighing approximately $1.5 \mathrm{~kg}$ were randomly divided into the control group (administered with an equal volume of normal saline), the semen litchi high dose group $(12.0 \mathrm{~g} / \mathrm{kg})$, the semen litchi medium dose group $(6.0 \mathrm{~g} / \mathrm{kg})$ and the semen litchi low dose group $(3.0 \mathrm{~g} / \mathrm{kg})$. Administration by gavage was conducted twice a day with an interval of 12 hours. Fasting without food (water allowed) was conducted 4 hours before administration for 3 consecutive days. One hour after the last administration by gavage, $3 \%$ sodium pentobarbital was used for anesthesia, and blood of the carotid artery was collected, left for 4 hours at $4^{\circ} \mathrm{C}$, centrifuged at 3000 $\mathrm{r} / \mathrm{min}$ for 15 minutes, and the serum was aseptically separated. After being inactivated at $56^{\circ} \mathrm{C}$ for 30 minutes, filtered and sterilised by $0.20 \mu \mathrm{m}$ microporous filtering membrane, semen litchi drug serum was prepared into the control group $(0 \mathrm{~g} / \mathrm{kg})$, high-dose group $(12.0 \mathrm{~g} / \mathrm{kg})$, medium-dose group $(6.0 \mathrm{~g} / \mathrm{kg})$ and low-dose group $(3.0 \mathrm{~g} / \mathrm{kg})$, and stored at $-20^{\circ} \mathrm{C}$ until use.

After HepG2 cells were resuscitated, they were cultured in DMEM nutrient solution (containing $10 \%$ calf serum, penicillin $100 \mathrm{U} / \mathrm{mL}$, and streptomycin $100 \mu \mathrm{g} / \mathrm{mL}$ ) in a $37^{\circ} \mathrm{C} 5 \% \mathrm{CO}_{2}$ incubator. The cells were adherent to the wall, each generation created in every 2-3 days, and cells in exponential growth phase were used for experiments.

Inhibitory effect of semen litchi drug serum on HepG2 cell proliferation was detected by MTT Assay. HepG2 cells in logarithmic growth phase were inoculated in a 96-well plate at a cell concentration of $3 \times 10^{4} / \mathrm{mL}, 100 \mu \mathrm{l}$ of each well. The cells were adherently grown for 24 hours before a final concentration of $30 \%$ of the drugcontaining serum medium acted on the cells. Four duplicate wells were set for the four groups of concentrations of the control group, the high dose group, the medium dose group and low dose group. The final concentration of $5 \mathrm{~g} / \mathrm{LMTT}$ solution was added after 72 hours of culture. After incubation for 4 hours in the incubator, the culture medium was removed. DMSO was added to dissolve the crystals. The absorbance at $\mathbf{5 7 0}$ $\mathrm{nm}$ was measured with a microplate reader to calculate the inhibitory effect of semen litchi drug serum on HepG2 cells after 24, 48 and 72 hours. Calculation formula: cell inhibition rate $(\%)=$ (absorbance optical density (OD) value of the control group - absorbance OD value of the treated group) / absorbance OD value of the control group $\times 100 \%$.

The expression of VEGF and MMP-9 mRNA in HepG2 Cells were detected by real-time PCR. According to the cell count results, the cell density was adjusted to $1 \times 10^{6}$ cells $/ \mathrm{mL}$, and concentrations of the control group, the high-dose group, the medium-dose group and the lowdose group acted on HepG2 cells for 48 hours, respectively. Total mRNA kit from Takara Company was used to extract total cellular RNA. cDNA was prepared as described in instruction of the Prime Script $₫ R T$ Kit with gDNA Eraser (Perfect Real Time) reverse transcription kit. Real-time PCR amplification was conducted using cDNA as template and GADPH as internal reference. The VEGF upstream primer: 5'TGGAGTGTGTGCCCACTGAG-3'; the downstream primer: 5'-TGCATTCACATTTGTGCTGTAG-3', and the amplified fragment length was $117 \mathrm{bp}$. MMP-9 upstream primer: 5'-TCCCTG-GAGACCTGAGAACC-3'; downstream primer: 5'-CG-GCAAGTCTTCCGAGTAGTTT-3', and the amplified fragment length was $307 \mathrm{bp}$. GAPDH upstream primer: 5'-GCACCGTCAAGGCTGAGAAC-3'; downstream primer: 5'-TGGTGAAGACGCCAGTGGA$3^{\prime}$, and the amplified fragment length was $138 \mathrm{bp}$. The reaction system was $2 \times$ super real pre mix plus $10 \mu \mathrm{L}$, $0.8 \mu \mathrm{L}$ of each of the upstream and downstream primers (concentration: $10 \mu \mathrm{mol} / \mathrm{L}$ ), $2 \mu \mathrm{L}$ of cDNA (stock solution), $1 \mu \mathrm{L}$ of ROX reference dye (50x), and $6.4 \mu \mathrm{L}$ of RNase free $\mathrm{dH}_{2} \mathrm{O}$. The total system was $20 \mu \mathrm{L}$. The reaction conditions were as follows: pre-denaturation at $95^{\circ} \mathrm{C}$, $30 \mathrm{~s}$ for one reaction cycle, $95^{\circ} \mathrm{C}$ for $10 \mathrm{~s}, 60^{\circ} \mathrm{C}$ for $34 \mathrm{~s}$, a total of 40 cycles; $55^{\circ} \mathrm{C}-95^{\circ} \mathrm{C}$ for $30 \mathrm{~s}$, a total of 81 reaction cycles. After the end of PCR amplification, the analyser displayed the standard curve, amplification curve and melting curve. The amplification factor was calculated using the2- $\triangle \triangle \mathrm{Ct}$ method.

The expression of VEGF and MMP-9 protein in HepG2 cells were detected by western blot. After treatment of HepG2 cells for 48 hours with concentrations of the control group, high-dose group, medium-dose group and low-dose group, strong RIPA (premixed with PMSG $100: 1)$ lysate was used to extract total protein of cells from each group. BCA kit was used to determine protein concentration. Twenty $\mu \mathrm{g}$ total protein was used to prepare protein sample for loading, $10 \%$ SDS-PAGE gel electrophoresis, PVDF membrane transfer, membrane closure for 2 hours, and primary antibody incubated overnight at $4^{\circ} \mathrm{C}$. The membrane was washed 3 times with TBST, and secondary antibody (HRP-labelled goat anti-mouse antibody) was added and incubated at room temperature for one hour. The membrane was washed three times (10 minutes/time) and exposed to light by chemiluminescence. $\beta$-actin was used as internal reference.

SPSS 22.0 software was used for statistical analysis of the data. Measurement data was expressed with mean $\pm S D$. One-way ANOVA analysis of measurement data was used for comparison among groups. The SNK test was used for the mutual comparison between multiple groups. The $p<0.05$ was considered to be statistically significant. 


\section{RESULTS}

The semen litchi low-dose group, medium-dose group, and high-dose group acted on the HepG2 cells for 24, 48 , and 72 hours, respectively. The results showed that the inhibitory effect of semen litchi drug serum on the proliferation of HepG2 cells significantly increased with the increase of drug concentration, which was dose-time dependent. The inhibitory rate of HePG2 cell proliferation in the semen litchi low-dose group, mediumdose group, and high-dose group was significantly different from that in the control group (all $p<0.001$, Table I).

After acting on HepG2 cells for 48 hours, the expression levels of VEGF and MMP-9 mRNA in HepG2 cells after 48 hours of treatment by semen litchi low-dose group, medium-dose group, and high-dose group were significantly lower than those in the control group (all $p<0.001$, Table II); and with the increase of semen litchi drug serum concentration, the inhibitory effect on the expression of VEGF and MMP-9 mRNA became more obvious.

After acting on HepG2 cells for 48 hours, the relative expressions of VEGF and MMP-9 protein in semen litchi low-dose group, medium-dose group, and high-dose group were lower than those in control group (all $p<0.001$ ); and with the increase of concentration, semen litchi drug serum's inhibition of VEGF, MMP-9 protein expression increased, as shown in Figure 1.

\section{DISCUSSION}

The use of medicine-containing serum instead of crude extracts as a medicine-carrier can effectively avoid the

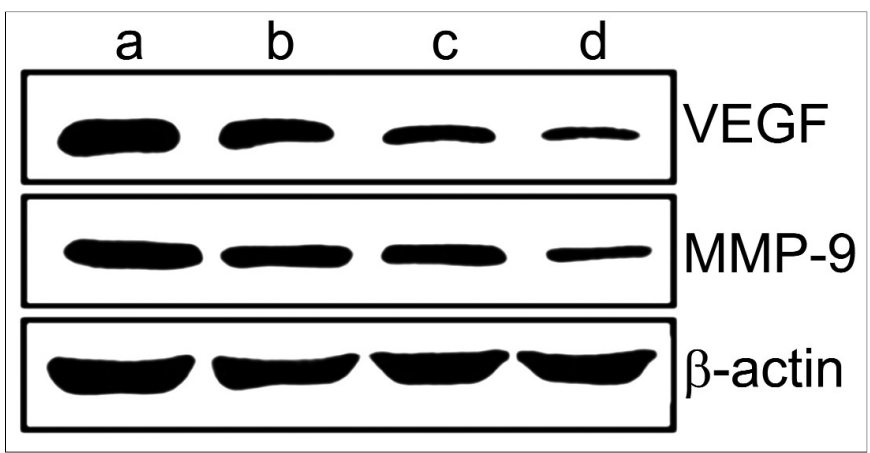

Figure 1: The effect of semen litchi drug serum on the expression of VEGF and MMP-9 in HepG2 cells after $48 \mathrm{~h}$ of treatment.

(a) Control group; (b) Semen litchi low-dose group; (c) Semen litchi mediumdose group; (d) Semen litchi high-dose group.
Table II: The effect of semen litchi drug serum on the expression of VEGF mRNA and MMP-9 mRNA in HepG2 cells after 48h of treatment $(n=4)$.

\begin{tabular}{l|cc|cc}
\hline \multirow{2}{*}{ Groups } & \multicolumn{2}{|c|}{ VEGF mRNA } & \multicolumn{2}{c}{ MMP-9 mRNA } \\
\cline { 2 - 5 } & Mean \pm SD & $p$-value & Mean \pm SD & $p$-value \\
\hline Control group & $0.972 \pm 0.163$ & $<0.001$ & $1.036 \pm 0.058$ & $<0.001$ \\
\hline $\begin{array}{l}\text { Semen litchi } \\
\text { low-dose group }\end{array}$ & $0.610 \pm 0.335$ & & $0.700 \pm 0.346$ & \\
\hline $\begin{array}{l}\text { Semen litchi } \\
\text { medium-dose group }\end{array}$ & $0.252 \pm 0.171$ & $0.401 \pm 0.230$ & \\
\hline $\begin{array}{l}\text { Semen litchi } \\
\text { high-dose group }\end{array}$ & $0.080 \pm 0.074$ & & $0.127 \pm 0.084$ & \\
\hline
\end{tabular}

disadvantages brought by direct use of crude extracts of traditional medicines, such as excessive ingredients, difficulty in determining active ingredients, inconsistent in vitro and in vivo test results and efficacies and many other problems. 9,10 Therefore, in vitro experiments using drug-containing serum can simulate the actual process of pharmacological effects produced by the agent in vivo, which is theoretically more scientific and authentic.

The occurrence of tumors is associated with abnormal cell proliferation and differentiation and the inhibition of its apoptosis.11,12 Inhibiting tumor proliferation and promoting tumor cell apoptosis are important methods for tumor prevention and treatment. Traditional Chinese medicine semen litchi has the effects of promoting the circulation of qi, dissolving lumps, resisting coldness and relieving pain. Studies have confirmed that semen litchi has many pharmacological effects. ${ }^{13}$ In addition, semen litchi has a high content of crude fiber, minerals, essential amino acids, and essential fatty acids, as well as litchi acid with a good protective effect on the cardiovascular system. It is a good source of nutrition for the development of elderly health foods. Studies have shown that litchi seed extracts had anti-prostate cancer effect. 14 Other research has shown that litchi seed extract could suppress epidermal growth factor receptor signalling and inhibited non-small cell lung cancer cell growth. 15

In this experiment, serum pharmacological methods were used to observe the effect of semen litchi drug serum on the proliferation inhibition rate of human hepatoma HepG2 cells. The results of this study indicate that semen litchi drug serum has a good effect on inhibiting proliferation of HepG2 cells, and showing a dose-time dependent effect. This conclusion was similar to the result of Wang et al., which indicated that litchi fruit pericarp extract demonstrated a dose- and timedependent inhibitory effect on cancer cell growth. ${ }^{16}$

Table I: The effect of semen litchi drug serum on the proliferation inhibition rate of HepG2 Cells $(n=4)$.

\begin{tabular}{|c|c|c|c|c|c|c|}
\hline \multirow[t]{2}{*}{ Groups } & \multicolumn{2}{|c|}{$24 \mathrm{~h}$} & \multicolumn{2}{|c|}{$48 \mathrm{~h}$} & \multicolumn{2}{|c|}{$72 \mathrm{~h}$} \\
\hline & OD value & Inhibitory rate $(\%)$ & OD value & Inhibitory rate $(\%)$ & OD value & Inhibitory rate (\%) \\
\hline Control group & $0.54 \pm 0.01$ & $0.00 \pm 0.00$ & $0.53 \pm 0.01$ & $0.00 \pm 0.00$ & $0.54 \pm 0.02$ & $0.00 \pm 0.00$ \\
\hline Semen litchi low-dose group & $0.50 \pm 0.05$ & $7.41 \pm 0.07$ & $0.49 \pm 0.01$ & $7.55 \pm 0.23$ & $0.49 \pm 0.01$ & $9.26 \pm 0.23$ \\
\hline Semen litchi medium-dose group & $0.47 \pm 0.02$ & $12.90 \pm 0.28$ & $0.44 \pm 0.02$ & $16.98 \pm 0.62$ & $0.44 \pm 0.01$ & $18.52 \pm 0.58$ \\
\hline Semen litchi high-dose group & $0.40 \pm 0.03$ & $25.93 \pm 0.69$ & $0.38 \pm 0.02$ & $28.30 \pm 1.15$ & $0.36 \pm 0.02$ & $33.33 \pm 1.39$ \\
\hline p-value & $<0.001$ & $<0.001$ & $<0.001$ & $<0.001$ & $<0.001$ & $<0.001$ \\
\hline
\end{tabular}


VEGF is the most potent angiogenic factor in vivo. It is highly expressed in liver cancer, and its receptors (VEGFRs) play an important role in the growth, metastasis and recurrence of liver cancer. The major reason for the difficulty of treating primary liver cancer is invasion and metastasis. Matrix metalloproteinases (MMPs) are a family of endopeptidases that degrade ECM. They play an important role in cell movement, basement membrane degradation and lumen formation during angiogenesis and are closely related to the invasion and metastasis of primary liver cancer. ${ }^{17}$ In addition, studies have shown that vascular endothelial growth factor stimulates endothelial cells to produce MMPs, or activates the matrix-degrading cascade reaction by modulating the balance of MMPs and their inhibitors tissue inhibitors of metalloproteinases (TIMPs), thus allowing tumor cells to penetrate more easily and affecting the infiltration and metastasis of tumors. 18,19 In the MMP family, MMP-9 is a common tumor marker that reflects the invasion, metastasis, and recurrence of primary liver cancer. 20 At present, the specific molecular mechanism of anti-hepatocarcinoma effect of semen litchi drug serum is not yet known.

The results showed that the expression levels of VEGF and MMP-9 mRNA and protein were lower in HepG2 cells treated with semen litchi drug serum in the low, medium and high dose groups than those in the control group, and with the increase of concentration, semen litchi drug serum's inhibition of the expression of VEGF and MMP-9 mRNA and protein increased. It suggested that the semen litchi drug serum had a certain inhibitory effect on the expression of VEGF and MMP-9 mRNA and protein. This confirmed the inhibitory effect of semen litchi drug serum on the expression of VEGF and MMP-9 in HepG2 cells from the level of gene and protein expression.

One study had shown that litchi seed extracts caused dose-dependent apoptosis of HepG2 cells through cell cycle arrest at G0/G1 phase, thus preventing cells entering $S$ or G2/M phase. ${ }^{21}$ In view of this, the authors speculated that semen litchi drug serum might inhibit cell proliferation by G0/G1 phase arrest in HepG2 cells. It could be further studied in the future to confirm it.

It is worth mentioning that, at present, studies on litchi anti-tumor have shown good anti-tumor activity only in vitro. The research and development of litchi antineoplastic drugs is still in the laboratory evaluation stage. There are no litchi antineoplastic drugs on the market, and they have yet to be further developed.

\section{CONCLUSION}

In short, semen litchi drug serum can inhibit the proliferation of hepatoma cells in vitro. The anti-hepatoma effect of semen litchi drug serum may be exerted through down-regulating the expression of VEGF and
MMP-9 and inhibiting angiogenesis of hepato-cellular carcinoma.

\section{REFERENCES}

1. Bae HJ, Jung KH, Eun JW, Shen Q, Kim HS, Park SJ, et al. Microrna-221 governs tumor suppressor HDAC6 to potentiate malignant progression of liver cancer. $J$ Hepatol 2015; 63: 408-19.

2. Murakami T, Tsurusaki M. Hypervascular benign and malignant liver tumors that require differentiation from hepatocellular carcinoma: Key points of imaging diagnosis. Liver Cancer 2014; 3:85-96.

3. Farinati F, Vanin V, Giacomin A, Pozzan C, Cillo U, Vitale A, et al. BCLC stage B hepatocellular carcinoma and transcatheter arterial chemoembolization: A 20 -year survey by the Italian liver cancer group. Liver Int 2015; 35:223-31.

4. Baba Y, Hayashi S, Ueno K, Nakajo M, Ueno S, Kubo F, et al. Comparison of survival rates between patients treated with transcatheter arterial chemoembolization and hepatic resection for solitary hepatocellular carcinoma. Oncol Lett 2010; 1:905-11.

5. Huang KW, Guo JW, Chen JM, Lin LJ, Xu F. Study on the chemical constituents in ethyl acetante extraction from semen litchi. J Chin Med Mater 2012; 35:64-6.

6. Li H, Zhou Z, Liu Q, Dong H, Duan Y, Li C, et al. Biological denitrification in high salinity wastewater using semen litchi as a carbon source. Rsc Adv 2015; 5:92836-42.

7. An FQ, Matsuda $M$, Fujii $H$, Matsumoto $Y$. Expression of vascular endothelial growth factor in surgical specimens of hepatocellular carcinoma. J Cancer Res Clin Oncol 2000; 126:153-60.

8. Yu D, Chen J, Sun X, Zhuang L, Jiang C, Ding Y. Mechanism of endothelial progenitor cell recruitment into neo-vessels in adjacent non-tumor tissues in hepatocellular carcinoma. BMC Cancer 2010; 10:435.

9. Jiang YR, Miao Y, Yang L, Xue M, Guo CY, Ma XJ, et al. Effect of Chinese herbal drug-containing serum for activating-blood and dispelling-toxin on ox-LDL-induced inflammatory factors' expression in endothelial cells. Chin J Integr Med 2011; 18: 30-3.

10. Cao Y, Liu F, Huang Z, Zhang Y. Protective effects of guanxin shutong capsule drug-containing serum on tumor necrosis factor- $\alpha$-induced endothelial dysfunction through nicotinamide adenine dinucleotide phosphate oxidase and the nitric oxide pathway. Exp Ther Med 2014; 8:998-1004.

11. Huang B, Zhou H, Lang X, Liu Z, Xiong F, Wang S. Expression of bag- 1 is closely related to cell differentiation and TNM stage in esophageal cancer and its downregulation inhibits the proliferation and invasion of human esophageal carcinoma cells. Oncol Rep 2014; 32:1441-6.

12. Yoon CJ, Chung JW, Park JH, Yoon YH, Lee JW, Jeong SY, et al. Transcatheter arterial chemoembolization with paclitaxellipiodol solution in rabbit VX2 liver tumor. Radiology 2003; 229:126-31.

13. Fei $H$, Zhang $R$, Yang $L$, Xiao J, Lei L, Wei Z, et al. Dietary litchi pulp polysaccharides could enhance immunomodulatory and antioxidant effects in mice. Int $J$ Biol Macromol 2016; 92: 1067-73. 
14. Guo H, Luo H, Yuan H, Xia Y, Shu P, Huang X, et al. Litchi seed extracts diminish prostate cancer progression via induction of apoptosis and attenuation of EMT through Akt/GSK-3 $\beta$ signaling. Sci Rep 2017; 7:41656.

15. Chung YC, Chen CH, Tsai YT, Lin CC, Chou JC, Kao TY, et al. Litchi seed extract inhibits epidermal growth factor receptor signaling and growth of two non-small cell lung carcinoma cells. BMC Complement Altern Med 2017; 17:16.

16. Wang X, Wei Y, Yuan S, Liu G, Zhang YL, Wang W. Potential anticancer activity of litchi fruit pericarp extract against hepatocellular carcinoma in vitro and in vivo. Cancer Lett 2006; 239:144-50

17. Shi F, Xiao F, Ding P, Qin H, Huang R. Long noncoding RNA highly upregulated in liver cancer predicts unfavorable outcome and regulates metastasis by MMPs in triple-negative breast cancer. Arch Med Res 2016; 47:446-53.
18. Groblewska M, Siewko M, Mroczko B, Szmitkowski M. The role of matrix metalloproteinases (MMPs) and their inhibitors (TIMPs) in the development of esophageal cancer. Folia Histochem Cytobiol 2012; 50:12-9.

19. Gentner B, Wein A, Croner RS, Zeittraeger I, Wirtz RM, Roedel F, et al. Differences in the gene expression profile of matrix metalloproteinases (MMPs) and their inhibitors (TIMPs) in primary colorectal tumors and their synchronous liver metastases. Anticancer Res 2009; 29:67-74.

20. Avadanei R, Caruntu ID, Amalinei C, Lozneanu L, Balan R, Grigoras A, et al. High variability in MMP2/TIMP2 and MMP9/TIMP1 expression in secondary liver tumors. Rom $\mathrm{J}$ Morphol Embryol 2013; 54:479-85.

21. Tao $X H$, Wang $H$, Wang $Y$, Huang XS, Guo GQ, Shen WZ. Effect of litchi seed extract on proliferation and apoptosis of HepG-2 in vitro. Nat Prod Res Dev 2008; 20:988-92. 